Signal Processing 54 (1996) 81-84

\title{
Repeated fractional Fourier domain filtering is equivalent to repeated time and frequency domain filtering
}

\author{
Haldun M. Ozaktas* \\ Department of Electrical Engineering, Bilkent University, 06533 Bilkent, Ankara, Turkey \\ Received 15 January 1996; revised 22 April 1996
}

\begin{abstract}
Any system consisting of a sequence of multiplicative filters inserted between several fractional Fourier transform stages, is equivalent to a system composed of an appropriately chosen sequence of multiplicative filters inserted between appropriately scaled ordinary Fourier transform stages. Thus every operation that can be accomplished by repeated filtering in fractional Fourier domains can also be accomplished by repeated filtering alternately in the ordinary time and frequency domains.
\end{abstract}

\section{Zusammenfassung}

Jedes System, bestehend aus einer Sequenz von multiplikativen Filtern, die zwischen mehrere fraktionale FourierTransformationsstufen eingefügt sind, ist äquivalent zu einem System, das aus einer geeignet gewählten Sequenz von multiplikativen Filtern zusammengesetzt ist, die zwischen passend skalierte normale Fourier-Transformationsstufen eingefügt werden. Auf diese Weise kann jede Operation, die durch wiederholte Filterung im fraktionalen Fourier-Bereich erzielt werden kann, ebenso durch wiederholte Filterung abwechselnd im normalen Zeit- und Frequenzbereich erzielt werden.

\section{Résumé}

Tout système consistant en une séquence de filtres multiplicatifs insérés entre plusieurs étages de transformation de Fourier fractionnaire est équivalent à un système composé d'une séquence correctement choisie de filtres multiplicatifs insérés entre des étages de transformation de Fourier échelonnés de manière appropriée. De ce fait, toute opération qui peut être accomplie par filtrage répété dans le domaine de Fourier fractionnaire peut aussi être effectuée en répétant le filtrage alternativement dans les domaines temporel et fréquentiel conventionnels.

Keywords: Time-variant filtering; Fractional Fourier transforms

*Tel: 90312266 4307; fax: 90312266 4126; e-mail: haldun@ee.bilkent.edu.tr. 
Let $x(t)$ denote the time, and $X(f)$ the frequency domain representation of an input signal. Conventional Fourier domain filtering involves multiplication of $X(f)$ with a filter function $H(f)$ to obtain the Fourier transform $Y(f)=H(f) X(f)$ of the filtered output signal. This type of filtering allows the realization of time-invariant (convolution type) linear operations only: $y(t)=h(t) * x(t)$. By defining the Fourier transform operator $\mathscr{F}$ and the multiplicative filter operator $A_{H}$, the relation between the input and output of the system can be expressed as

$y=\mathscr{F}^{-1} \Lambda_{H} \mathscr{F} x$.

By interpreting $x, y$ as signal vectors, $\mathscr{F}$ as the DFT matrix, and $\Lambda_{H}$ as a diagonal matrix, the above expression can also be interpreted in a discrete-time setting.

So-called generalized filtering systems were proposed in previous work on the fractional Fourier transform $[8,9]$. These systems involve multiplicative filters inserted between several fractional Fourier transform stages (Fig. 1(a)). Each fractional Fourier transform stage transforms from one fractional domain to another, where a multiplicative filter is applied. In other words, the signal is repeatedly filtered in several consecutive fractional Fourier domains. It was shown that this allows the realization of certain time-variant operations. In operator notation, a system involving $M$ stages can be expressed as

$y=\Lambda_{I I_{M+1}} \mathscr{F}^{a_{M}} \Lambda_{I I_{M}} \cdots \Lambda_{I I_{3}} \mathscr{F}^{a_{2}} \Lambda_{H_{2}} \mathscr{F}^{a_{1}} \Lambda_{H_{1}} \mathscr{F}^{a_{0}} \Lambda_{I I_{0}} x$,

where $\mathscr{F}^{a}$ is the ath order fractional Fourier transform operator.

In its most general form, the fractional Fourier transform operation $[6,9,1]$ has three parameters: the order $a$, the input scale parameter $s_{\text {in }}$, and the output scale parameter $s_{\text {out }}[11]$. (When we set $s_{\text {in }}$ and $s_{\text {out }}$ equal to unity, we recover the pure mathematical form of the transform.) The ath order fractional Fourier transform is denoted by $x_{a}(t)$ and is given by

$$
\begin{aligned}
& x_{a}(t)=\int_{-\infty}^{\infty} h\left(t, t^{\prime}\right) x\left(t^{\prime}\right) \mathrm{d} t^{\prime}, \\
& h\left(t, t^{\prime}\right)=C \exp \left[\mathrm { i } \pi \left(\cot \phi \frac{t^{2}}{s_{\text {out }}^{2}}-2 \operatorname{cosec} \phi \frac{t t^{\prime}}{s_{\text {in }} s_{\text {out }}}\right.\right. \\
& \\
&\left.\left.+\cot \phi \frac{t^{\prime 2}}{s_{\text {in }}^{2}}\right)\right],
\end{aligned}
$$

(a)

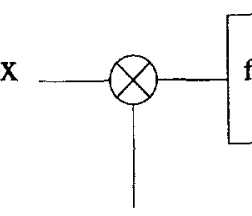

$\mathrm{H}_{0}$
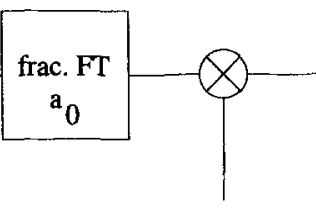

$\mathrm{H}_{1}$

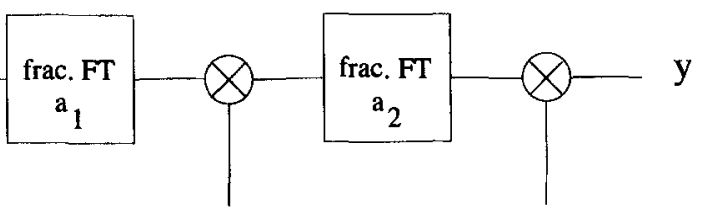

$\mathrm{H}_{2}$

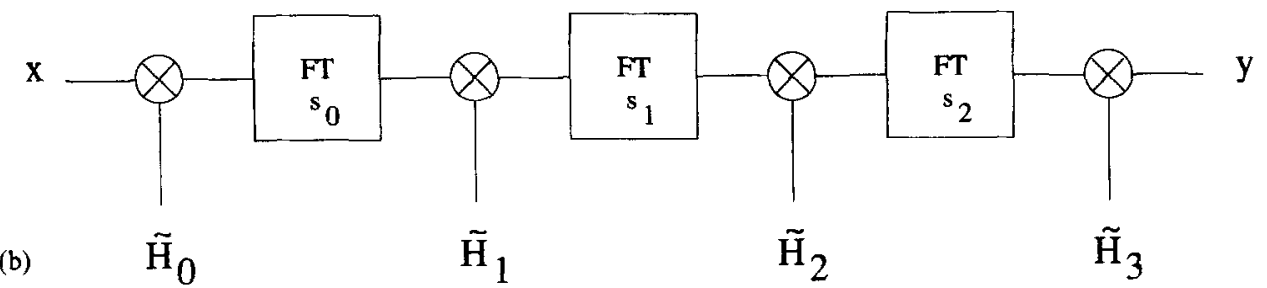

Fig. 1. (a) A sequence of multiplicative filters inserted between fractional Fourier transform stages, each of which is characterized by its order $a_{j}$. The scale parameter $s$ is the same for all stages. (b) The system modeled as a sequence of multiplicative filters inserted between ordinary Fourier transform stages, each of which is characterized by its scale parameter $s_{j}$. 
where $\phi=a \pi / 2$, and $C$ depends on $a, s_{\text {in }}$ and $s_{\text {out }}$ in a manner that is not relevant for our purposes. When $a$ is an even or odd multiple of 2 , the kernel $h\left(t, t^{\prime}\right)$ approaches $\delta\left(t-t^{\prime}\right)$ or $\delta\left(t+t^{\prime}\right)$, respectively. The ordinary Fourier transform is obtained when we set $a=1$. In this paper it will be sufficient to employ fractional transforms whose input and output scale parameters are equal $\left(s \equiv s_{\text {in }}=s_{\text {out }}\right)$. The ath fractional Fourier domain is defined by the axis which makes angle $\phi=a \pi / 2$ with the $t$ axis in the time-frequency plane [12]. Fractional Fourier transforms can be realized optically $[5,8,11]$, or digitally in $\mathrm{O}(N \log N)$ time $[3,13]$.

In this paper we show that any system of the form defined by Eq. (2) (Fig. 1(a)) is equivalent to a system composed of filters inserted between ordinary Fourier transform stages, appropriately scaled (Fig. 1(b)). Each time a Fourier transform is applied we alternate between the time and frequency domains, where multiplicative filters are applied. In operator notation,

$y=\Lambda_{\tilde{H}_{M+1}} \mathscr{F}_{s_{M}} \Lambda_{\tilde{H}_{M}} \cdots \Lambda_{\hat{H}_{3}} \mathscr{F}_{s_{2}} \Lambda_{\tilde{H}_{2}} \mathscr{F}_{s_{1}} \Lambda_{\tilde{H}_{1}} \mathscr{F}_{s_{0}} \Lambda_{\tilde{H}_{0}} x$,

where $\mathscr{F}_{s}$ is the scaled ordinary Fourier transform operator with associated kernel

$h\left(t, t^{\prime}\right)=\exp \left(-i 2 \pi t t^{\prime} / s^{2}\right)$.

The claim is that by appropriate choice of filters $\tilde{H}_{j}$ and scale factors $s_{j}$, this relation between $x$ and $y$ can be made the same as that given in Eq. (2).

The proof is elementary. Upon examining the kernel of the fractional Fourier transform, we observe that calculating the fractional transform $x_{a}(t)$ amounts to multiplying $x(t)$ by a chirp function, taking its scaled ordinary Fourier transformation, and multiplying the result by another chirp function. (It is important to note that whereas this approach serves the purpose of the present paper, it is not necessarily the best way of decomposing the transform for the purpose of digital computation [13].) The pre and post chirp multiplications can be absorbed into the multiplicative filters preceding and following the fractional transform stage, leaving us with a scaled ordinary Fourier transform.

This result can be easily generalized. We just argued that a fractional Fourier transform of any order can be reduced to a scaled ordinary Fourier transform. The ordinary Fourier transform is no more privileged than fractional transforms of other orders (nor easier or cheaper to implement). A transform of any fractional order can be likewise reduced to an appropriately scaled fractional transform of any other desired order (as easily demonstrated by manipulating Eq. (4)). Thus, by appropriate choice of scale factors and multiplicative filters, repeated filtering in any given sequence of fractional domains can be made equivalent to repeated filtering in any other desired sequence of fractional domains. In particular, we can choose to alternate between any given two domains. That is, applying multiplicative filters alternately in any two prespecified domains (provided their orders do not differ by an integer multiple of 2), allows us to do everything that can be done by any configuration of the form given in Fig. 1(a).

It is also possible to show that the scale factors $s_{j}$ appearing in Eq. (5) can be eliminated or made equal to each other. Since the Fourier transform of a scaled function is a scaled version of its Fourier transform, these scale factors can be migrated through the filters and transform stages and collected at either end of the system (by also replacing the filters with their appropriately scaled versions).

Let us now reiterate our main result. Generalized filtering systems employing fractional transforms (as in Fig. 1(a)) can be reduced to generalized filtering systems employing only the ordinary Fourier transform (as in Fig. 1(b)). Applying multiplicative filters alternately in the time and frequency domains allows us to do everything that can be done by applying filters in fractional Fourier domains.

This result does not compromise the conceptual and practical utility of the fractional Fourier transform. The fractional transform may be conceptually indispensable in devising an algorithm or designing an effective filter, even if the system is then reduced to one which does not employ fractional Fourier transforms. (Convincing examples of the utility of the fractional Fourier transform in designing filtering systems may be found in $[9,10$, $2,7,3,4]$.) Furthermore, one would not necessarily engage in such a reduction, since computation of the fractional transform - both optically and digitally - is not more difficult than computation of 
the ordinary transform. The computation of ordinary and fractional transforms can both be reduced to each other. The implementation of Fig. 1(a) is not more difficult than that of Fig. 1(b).

From a practical viewpoint, the implementation of the necessary filters may be much easier in certain domains, as compared to other domains (including the ordinary Fourier domain) where the filters may be difficult to realize. For instance, in chirp elimination $[10,2]$, the multiplicative filters necessary in fractional domains are simple binary pass/stop filters, whereas in the ordinary time and Fourier domains they would have to be complex functions. Furthermore, the accuracy needed to implement a filter in one domain may be less than in others. In conclusion, the equivalence results brought forward in this paper should be used to increase the number of alternative realizations which are nominally equivalent, not to reduce them to one. These alternative realizations provide additional degrees of freedom which may allow us to deal effectively with certain practical and technical constraints arising from sampling and quantization.

\section{References}

[1] L.B. Almeida, "The fractional Fourier transform and time-frequency representations", IEEE Trans. Signal Process., Vol. 42, 1994, pp. 3084-3091.
[2] R.G. Dorsch, A.W. Lohmann, Y. Bitran, D. Mendlovic and H.M. Ozaktas, "Chirp filtering in the fractional Fourier domain”, Appl. Opt., Vol. 33, 1994, pp. 7599-7602.

[3] M.A. Kutay, H.M. Ozaktas, L. Onural and O. Arikan, "Optimal filtering in fractional Fourier domains", Proc. 1995 IEEE Internat. Conf. Acoust. Speech Signal Processing, Detroit, Michigan, May 1995, pp. 937-940.

[4] M.A. Kutay, H.M. Ozaktas, O. Arikan and L. Onural, "Optimal filtering in fractional Fourier domains", IEEE Trans. Signal Process., Submitted.

[5] A.W. Lohmann, "Image rotation, Wigner rotation and the fractional Fourier transform", J. Opt. Soc. Amer. A, Vol. 10, 1993, pp. 2181-2186.

[6] A.C. McBride and F.H. Kerr, "On Namias's fractional Fourier transform”, IMA J. Appl. Math., Vol. 39, 1987, pp. 159-175.

[7] D. Mendlovic, H.M. Ozaktas and A.W. Lohmann, "Fractional correlation”, Appl. Opt., Vol. 34, 1995, pp. 303-309.

[8] H.M. Ozaktas and D. Mendlovic, "Fractional Fourier transformations and their optical implementation: Part II", J. Opt. Soc. Amer. A, Vol. 10, 1993, pp. 2522-2531.

[9] H.M. Ozaktas, B. Barshan, D. Mendlovic and L. Onural, "Convolution, filtering, and multiplexing in fractional Fourier domains and their relation to chirp and wavelet transforms", J. Opt. Soc. Amer. A, Vol. 11, 1994, pp. 547-559.

[10] H.M. Ozaktas, B. Barshan and D. Mendlovic, "Convolution and filtering in fractional Fourier domains", Opt. Rev., Vol. 1, 1994, pp. 15-16.

[11] H.M. Ozaktas and D. Mendlovic, "Fractional Fourier optics", J. Opt. Soc. Amer. A, Vol. 12, 1995, pp. 743-51.

[12] H.M. Ozaktas and O. Aytür, "Fractional Fourier domains", Signal Processing, Vol. 46, No. 1, September 1995, pp. 119-124.

[13] H.M. Ozaktas, O. Arikan, M.A. Kutay and Gozde Bozdagi, "Digital computation of the fractional Fourier transform and the discrete fractional Fourier transform", IEEE Trans. Signal Process., 1996, To appear. 